\title{
ARTICLE
}

\section{Luminescence properties and Crystal structures of Pr-doped GPS}

\author{
Youichi TSUBOTA ${ }^{1, *}$, Junichi H. KANEKO ${ }^{1}$, Mikio HIGUCHI ${ }^{1}$, Sohan KAWAMURA ${ }^{1,3}$, Shusuke NISHIYAMA ${ }^{1}$, \\ Fumiyuki FUJITA $^{1}$, Shunsuke UEDA ${ }^{2}$, Kazuhisa KURASHIGE ${ }^{2}$, Hiroyuki ISHIBASHI ${ }^{2}$, Akira HOMMA ${ }^{1}$ \\ and Michihiro FURUSAKA ${ }^{1}$
}

${ }^{1}$ Graduate School of Engineering, Hokkaido University N13, W8, Kita-ku, Sapporo 060-8628 Japan

${ }^{2}$ Hitachi Chemical Ltd., 1380-1 Tarazaki, Hitachinaka-shi 305-0047, Japan.

${ }^{3}$ present address: NTT Photonics Laboratories, Morinosato Wakamiya, Atsugi-shi, Kanagawa, 234-0198 Japan

\begin{abstract}
Crystal structures and scintillation properties of praseodymium-doped gadolinium pyrosilicate $\left(\mathrm{Gd}_{2} \mathrm{Si}_{2} \mathrm{O}_{7}: \operatorname{Pr}\right.$, GPS:Pr) were investigated as a part of systematic studies of $\mathrm{a}_{2} \mathrm{Si}_{2} \mathrm{Si}_{2} \mathrm{O}_{7}$ system as scintillation materials. Transparent yellowish-green polycrystalline GPS:Pr was grown using the floating zone growth method. The crystal structure, which is strongly related with the mean ionic radius of rare-earth elements, was orthorhombic with Pna2 ${ }_{1}$ space group for 5 and $10 \mathrm{~mol} \%$ Pr concentration. It was triclinic with $\mathrm{P} / 1$ space group for $15 \mathrm{~mol} \%$ and $20 \mathrm{~mol} \% \mathrm{Pr}$ concentration. A fluorescence spectrum was composed of a peak located at $300 \mathrm{~nm}$ and a broad peak around $360 \mathrm{~nm}$. Light yield of ca. $80 \%$ of a GSO:Ce scintillator was confirmed with GPS: $\operatorname{Pr}(\mathrm{Pr}=10 \mathrm{~mol} \%$ ) for $662 \mathrm{keV}$ gamma rays; the decay time was 26 ns. Unexpectedly, GPS:Pr with a triclinic structure, i.e. $\mathrm{Pr}=15$ and $20 \mathrm{~mol} \%$, showed no scintillation for gamma rays.
\end{abstract}

\section{KEY WORDS: $\mathrm{Gd}_{2} \mathrm{Si}_{2} \mathrm{O}_{7}$, scintillators, crystal structure, decay time, light output}

\section{Introduction}

In the past several decades, inorganic scintillators with high light yield, high detection efficiency, and fast decay time have been sought in the fields of nuclear medical diagnostics systems, high-energy physics, and others ${ }^{1), 2}$. To obtain higher detection efficiency, a scintillation material with a large product of density and the fourth power of an effective atomic number, $\rho Z_{\text {eff }}^{4}$, is necessary. Consequently, extensive studies have specifically examined rare-earth oxide materials. Moreover, aiming at large light yield and fast decay time, investigations of effective activators have also been conducted. Consequently, high-performance rare-earth oxide scintillators using $\mathrm{Ce}^{3+}$ activator, i.e., GSO:Ce, LSO:Ce etc. have been developed ${ }^{3,4), 5}$. A recently developed $\mathrm{LaBr}_{3}$ scintillator with 1.5 times greater light yield than that of a conventional NaI:Tl scintillator ${ }^{6}$ also uses $\mathrm{Ce}^{3+}$ as an activator.

As a promising newly developed scintillator, a cerium doped gadolinium pyrosilicate exists: $\mathrm{Gd}_{2} \mathrm{Si}_{2} \mathrm{O}_{7}: \mathrm{Ce}$ (GPS:Ce). First, GPS:Ce was investigated as a neutron scintillator for neutron scattering experiments because of its extremely large neutron capture cross section of $\mathrm{Gd}^{7)}$. This scintillator had a five-times-greater light yield than conventional BGO scintillator and a decay time of $46 \mathrm{~ns}$. Furthermore, a GPS:Ce scintillator had a value of $\rho Z_{\text {eff }}{ }^{4}$ higher than that of NaI:Tl and $\mathrm{LaBr}_{3}: \mathrm{Ce}$. Moreover, the GPS:Ce showed no deliquescence and no self-activity. Therefore, development of a large single crystal of GPS:Ce is underway, aimed at applications for gamma rays measurement ${ }^{8), 9}$.

Rare-earth pyrosilicates have polymorphism according to a mean ionic radius containing rare-earth elements ${ }^{10), 11)}$. Consequently, crystal structures and luminescence properties were changed on amounts of rare-earth activators, i.e. cerium.

*Corresponding Author, E-mail:y_tsubota@eng.hokudai.ac.jp Tel./fax: +81117066678

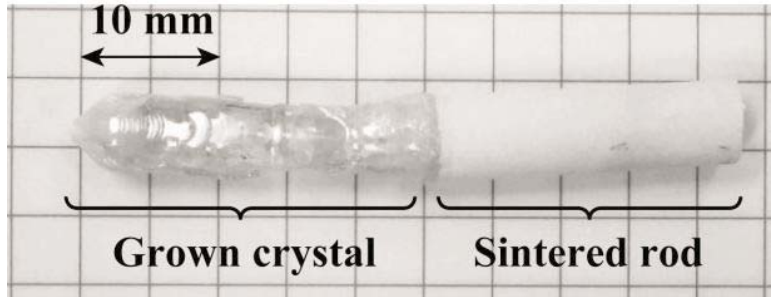

Fig. 1 Pr-doped GPS polycrystals grown using the floating zone method. Yellowish-green transparent crystals were obtained. Polycrystals were usually composed of single crystals of several millimeters.

For cerium-doped GPS, orthorhombic, triclinic, and other structures appeared depending on the cerium concentration; two peaks of the light yield occurred at cerium concentrations of $2.5 \mathrm{~mol} \%$ and $15 \mathrm{~mol} \%{ }^{12)}$.

For lutetium pyrosilicates (LPS), Pidol et al. investigated scintillation properties using $\mathrm{Ce}^{3+}, \mathrm{Pr}^{3+}$, and $\mathrm{Nd}^{3+}$ activators. The Pr-doped LPS had a fast decay time of $15 \mathrm{~ns}^{13)}$. Transitions of $\mathrm{Pr}^{3+}$ and $4 \mathrm{f}^{\mathrm{n}}-4 \mathrm{f}^{\mathrm{n}-1} 5 \mathrm{~d}^{1}$ have an energy band gap that is $1.5 \mathrm{eV}$ wider than that of $\mathrm{Ce}^{3+}$ in the same host ${ }^{14)}$. In general, a rough correlation exists between the emission wavelength and transition rate ${ }^{15}$; $\mathrm{Pr}^{3+}$-doped compounds have been investigated as fast scintillators ${ }^{16}$.

Crystal structures and scintillation properties of Pr-doped GPS crystals were investigated for systematic studies of the $\mathrm{Gd}_{2} \mathrm{Si}_{2} \mathrm{O}_{7}$ system as a scintillation material. The praseodymium concentration was $5-20 \mathrm{~mol} \%$ in this study.

\section{Experimental}

\section{Crystal Growth}

For this study, GPS:Pr crystals were grown using a floating zone (FZ) growth method. Starting materials were powders 
of $\mathrm{Gd}_{2} \mathrm{O}_{3}, \mathrm{SiO}_{2}$ with $99.999 \%$ purity and $\mathrm{Pr}_{6} \mathrm{O}_{11}$ with $99.99 \%$ purity. These materials were weighed and mixed at compositions of $\left(\mathrm{Gd}_{1-\mathrm{x}} \mathrm{Pr}_{\mathrm{x}}\right)_{2} \mathrm{Si}_{2} \mathrm{O}_{7}(x=0.05,0.10,0.15,0.20)$. Then the mixed powders were pressed isostatically $(60 \mathrm{MPa})$ into 5 $\mathrm{mm}$ diameter rods and sintered at $1650^{\circ} \mathrm{C}$ for $8 \mathrm{hr}$ in air.

These sintered rods were fixed on upper and lower shafts of a four mirror image furnace (FZ-T-10000-H-III-P-TK; Crystal Systems Inc., Yamanashi, Japan) for which four halogen lamps with maximum power of $750 \mathrm{~W}$ were used as a heating source. Crystal growth procedures were taken in a reduction atmosphere in $\mathrm{N}_{2}$, with a growth rate of $2 \mathrm{~mm} / \mathrm{h}$ and a counter-rotation rate of $15 \mathrm{rpm}$. Figure.1 presents an example of Pr-doped GPS polycrystals grown in this study; crystals were yellowish-green and transparent. The color deepened with increased praseodymium concentration.

Parts of the Pr-doped GPS polycrystalline crystals were cut into $2 \mathrm{~mm}$ thickness using a diamond cutter; then both sides of these samples were mechanically polished. A typical size of evaluated GPS:Pr samples was $3 \times 3 \times 2 \mathrm{~mm}$. Although size differences were found, the evaluated samples consisted of one or a few pieces of single crystals. Because of the lack of color uniformity, the distribution of $\operatorname{Pr}^{3+}$ was not observed in any samples. These samples were used for photoluminescence and scintillation property measurements.

\section{Crystal structure analysis by XRD}

Crystal structures of grown crystals were identified using $\mathrm{X}$-ray powder diffraction (XRD). Crystals were ground into powder using an agate mortar and pestle. The powder sam-

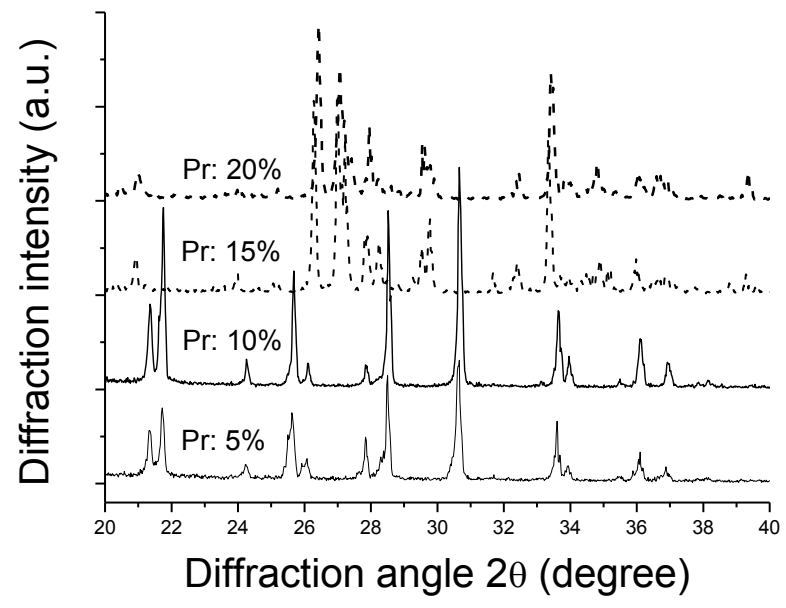

Fig. 2 X-ray diffraction patterns obtained with Pr-doped GPS crystals; concentrations of praseodymium were, respectively, 5, $10,15,20 \mathrm{~mol} \%$. For Pr concentrations of 5 and $10 \mathrm{~mol} \%$, a crystal structure was orthorhombic with the $\mathrm{Pna} 2_{1}$ space group. For $\mathrm{Pr}$ concentrations of 15 and $20 \mathrm{~mol} \%$, it was triclinic with the $\mathrm{P} / 1$ space group ples were measured using a powder X-ray diffractometer (JDX-3500; JEOL, Tokyo, Japan) with a $\mathrm{Cu}$ X-ray target with bias voltage of $30 \mathrm{kV}$ and beam current of $20 \mathrm{~mA}$. The obtained X-ray patterns were compared to data of the Joint Committee on Powder Diffraction Standards (JCPDS) to determine crystal structures.

\section{Photoluminescence and scintillation property mea- surements}

Excitation and emission spectra of photoluminescence were measured at room temperature using a spectrometer (FP6500; Jasco Inc., Tokyo, Japan). Wavelengths of excitation were $220-330 \mathrm{~nm}$. The measurement range of wavelengths for emission spectroscopy was 300-700 nm.

For scintillation property measurements, gamma rays of $662 \mathrm{keV}$ from a ${ }^{137} \mathrm{Cs}$ source were used. A GPS:Pr sample was set on a photomultiplier tube (H7195; Hamamatsu Photonics K.K., Shizuoka, Japan) using optical grease. As a reflector, a polytetrafluoroethylene tape was used. Output signals from the photomultiplier tube were connected to a delay line amplifier (460; Ortec, Oak Ridge, U.S.A.) for spectroscopy; the integral time of $0.1 \mu$ s was chosen. A linear gate and stretcher (542; Ortec, Oak Ridge, U.S.A.) and a multichannel analyzer (WE7562; Yokogawa, Tokyo) were used. In addition, output signals were measured using a digital oscilloscope with analog bandwidth of $1 \mathrm{GHz}$ (LT584; Lecroy Corp., New York, U.S.A.).

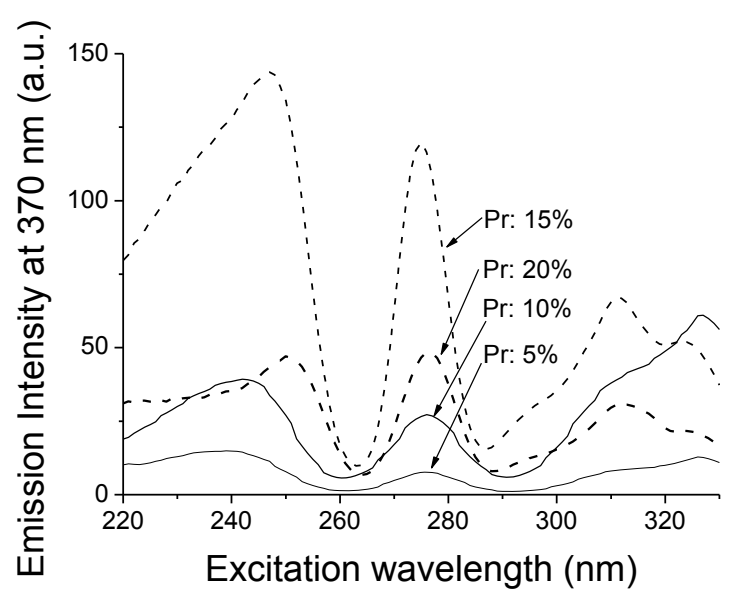

Fig. 3 Photoluminescence excitation spectra obtained with the Pr-doped GPS crystals. The wavelength of emission light for monitoring was set at $370 \mathrm{~nm}$. The peaks located at $275 \mathrm{~nm}$ correspond to transitions from $4 \mathrm{f}^{2}$ ground state to the $4 \mathrm{f}^{1} 5 \mathrm{~d}^{1}$ state of $\operatorname{Pr}^{3+}$ in the host lattice. 


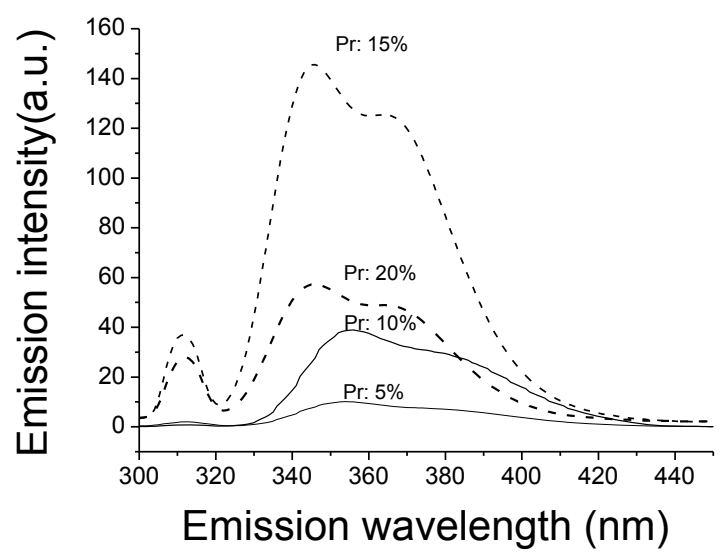

Fig. 4 Photoluminescence emission spectra obtained with the Pr-doped GPS crystals. The excitation wavelength was set at $275 \mathrm{~nm}$. Emission spectra were classified as one of two types according to their crystal structure: triclinic or orthorhombic structures

\section{Experimental results and Discussion}

\section{Change of crystal structures of GPS according to the praseodymium concentration}

As described in the Introduction, a crystal structure of rare-earth pyrosilicates is governed by a mean ionic radius containing rare-earth elements. Figure.2 portrays X-ray diffraction patterns obtained with Pr-doped GPS crystals; the $\mathrm{Pr}$ concentrations were $5,10,15$, and $20 \mathrm{~mol} \%$, respectively. For Pr concentrations of 5 and $10 \mathrm{~mol} \%$, a crystal structure was orthorhombic with Pna2 1 space group. For Pr concentrations of 15 and $20 \mathrm{~mol} \%$, it was triclinic with $\mathrm{P} / 1$ space group. The crystal structures obtained in this study were almost consistent with those described in an earlier report ${ }^{10)}$.

\section{Photoluminescence and scintillation properties}

Excitation and emission spectra were measured and classified as two types according to their crystal structures, orthorhombic for Pr concentration of 5 and $10 \mathrm{~mol} \%$, and triclinic for Pr concentration of 15 and $20 \mathrm{~mol} \%$. Scattering occurred in the size of the evaluated GPS samples. It is therefore difficult to compare the excitation and emission intensity quantitatively from these results.

The relation between excitation and emission spectra of photoluminescence was measured first. According to this result, the most intense emission wavelength, i.e. $370 \mathrm{~nm}$, was chosen for excitation spectrum measurements.

Figure. 3 shows the dependence of emission yield on the excitation wavelength obtained using Pr-doped GPS crystals. The excitation spectra include peaks located at $275 \mathrm{~nm}$ and broad peaks around 240 and $310 \mathrm{~nm}$. The peaks around 240 $\mathrm{nm}$ and at $275 \mathrm{~nm}$ probably correspond to transitions from the $4 \mathrm{f}^{2}$ ground state to the $4 \mathrm{f}^{1} 5 \mathrm{~d}^{1}$ state of $\mathrm{Pr}^{3+}$ in the host lat- tice ${ }^{[16]}$. However, the ratio between light yield of peaks at $250 \mathrm{~nm}$ and $275 \mathrm{~nm}$ increased concomitantly with increased concentration of praseodymium. In addition, assuming that the peaks around $240 \mathrm{~nm}$ consisted of two peaks, the peaks were, respectively, $230 \mathrm{~nm}$ and $250 \mathrm{~nm}$. In the case of the triclinic GPS, the intensity of the peak around $240 \mathrm{~nm}$ was higher than that obtained with the orthorhombic GPS; it was probably caused by a change in the crystal structure.

Figure.4 portrays emission spectra obtained from the Pr-doped GPS crystals; the excitation wavelength was set at $275 \mathrm{~nm}$. Peaks located at $310 \mathrm{~nm}$ were only observed for spectra obtained with the triclinic GPS. In addition, the tric-

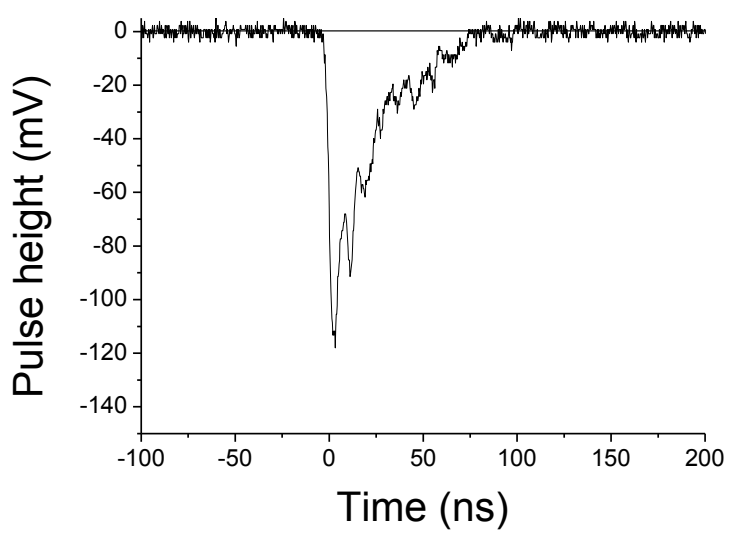

Fig. 5 An example of typical output signals from the orthorhombic GPS with Pr concentration of $10 \%$. This output signal was obtained using a H7195 photomultiplier and gamma rays of $662 \mathrm{keV}$ from a ${ }^{137} \mathrm{Cs}$ checking source. The average decay time was ca. $26 \mathrm{~ns}$.

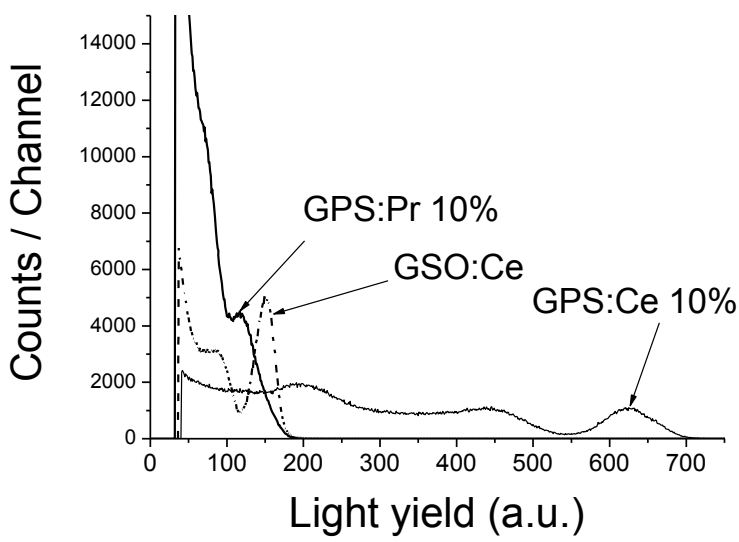

Fig. 6 Comparison of light yields of three scintillators: a commercially available $1.0 \mathrm{~mol} \%$ Ce-doped GSO, a $10 \mathrm{~mol} \%$ Ce-doped GPS, and the $10 \mathrm{~mol} \%$ Pr-doped GPS for $662 \mathrm{keV}$ gamma rays; the $10 \mathrm{~mol} \% \mathrm{Ce}$-doped GPS was triclinic. Although the Ce-doped GPS has ca. 4.1 times higher light yield than the GSO scintillator, the Pr-doped GPS was ca. 0.8 times light yield compared to GSO. 
linic GPS showed strong emission at 320-420 nm; peaks were located, respectively, at 340 and $365 \mathrm{~nm}$. However, considering the scattering of the sample sizes, the triclinic GPS had light yield larger than that of the orthorhombic GPS The orthorhombic GPS showed emissions of 330-420 nm; peaks were located at 350 and $380 \mathrm{~nm}$. These peaks were shifted to a longer wavelength than those with the triclinic GPS.

An example of typical output signals from the orthorhombic GPS with Pr concentration of $10 \%$ is presented in Fig. 5 . This output signal was obtained with the photomultiplier and gamma rays of $662 \mathrm{keV}$ from a ${ }^{137} \mathrm{Cs}$ checking source. An average decay time of the orthorhombic GPS with Pr concentration of $10 \%$ was 26 ns. Although the triclinic GPS showed strong emission light yield in the photoluminescence measurement, they had no response to gamma rays. A possibility is that emission light from the Pr-doped triclinic GPS has very slow decay time. However, no signal was observed by oscilloscope with longer time division up to $0.1 \mathrm{~s}$. Another possibility is that some inefficient energy transfer from the host lattice to $\operatorname{Pr}^{3+}$ activators occurred. Further investigation of the response of the triclinic GPS to gamma rays is necessary.

Figure.6 presents a comparison of light yields of three scintillators: a commercially available $1.0 \mathrm{~mol} \%$ Ce-doped GSO, a $10 \mathrm{~mol} \%$ Ce-doped GPS, and the $10 \mathrm{~mol} \%$ Pr-doped GPS for $662 \mathrm{keV}$ gamma rays; the $10 \mathrm{~mol} \% \mathrm{Ce}$-doped GPS was triclinic. Although the Ce-doped GPS has ca. 4.1 times higher light yield than the GSO scintillator, the Pr-doped GPS had ca. 0.8 times the light yield that GSO had.

In this comparison, Pr-doped GPS crystal have some cracks and strongly colored. Consequently, the full absorption peak has broad peaks for the Pr-doped GPS scintillator. From the perspective of light yield and energy resolution, further investigation using crack-free single crystals is necessary.

\section{Conclusion}

Luminescence properties and crystal structures of Pr-doped GPS scintillator were investigated. For $10 \mathrm{~mol} \% \mathrm{Pr}$-doped GPS, fast decay time of 26 ns was achieved. Although the triclinic GPS had a higher light yield than the orthorhombic GPS in photoluminescence spectroscopy, it showed no response to gamma rays.

To develop a higher performance scintillator, a scintillation mechanism of GPS scintillator must be clarified. Consequently, a systematic study and further understanding on a GPS system including Pr-doped GPS is necessary. In addi- tion, from the perspective of application, the Pr-doped GPS had 0.8 times the light yield of a GSO scintillator. It had fast decay time of $26 \mathrm{~ns}$ with neither self-radioactivity nor deliquescence. Therefore, a Pr-doped GPS can be a practically useful scintillator. Further investigations of Pr-doped GPS scintillators using large single crystals of $10 \mathrm{~mm}$ are indispensable to their development.

\section{Acknowledgment}

This study was partly supported by the Industrial Technology Research Grant Program in 2006 from the New Energy and Industrial Technology Development Organization (NEDO) of Japan.

\section{References}

1) C. W. E. van Eijk, Phys. Med. Biol., vol. 47, 2002, pp. R85-R106.

2) R. Boellaard, et al., Phys. Med. Biol. 48 (2003) 429.

3) K. Takagi and T. Fukuzawa, Appl. Phys. Lett., vol. 42, no. 1, 1983, pp. 43-44.

4) J.D. Naud, T.A. Tombrello, et al., IEEE Trans. Nucl. Sci. 43 (1995) 1324.

5) K. Takagi and T. Fukuzawa, "Cerium-activated Gd2SiO5 single crystal scintillator," Appl. Phys. Lett., vol. 42, no. 1, 1983, pp. 43-44.

6) W.M. Higgins, A. Churilov, E. van Loef, J. Glodo, M. Squillante, K. Shah, Journal of Crystal Growth 310 (2008) 2085-2089.

7) J. Haruna, et al., "Response function measurement of $\mathrm{Gd}_{2} \mathrm{Si}_{2} \mathrm{O}_{7}$ scintillator for neutrons," in preparation.

8) S. Kawamura, et al., Nuclear Instruments and Methods in Physics Research A 583 (2007) 356-359.

9) S. Kawamura, et al., IEEE TRANSACTIONS ON NUCLEAR SCIENCE, VOL. 54, NO. 4, AUGUST 2007.

10) J.Felsche, Journal of the Less-Common Metals, 21 (1970) 1-14.

11) N. Maiera, G. Rixeckerb,1, K.G. Nickel, Journal of Solid State Chemistry 179 (2006) 1630-1635.

12) S. Saeki, et al, "Dependence of crystal structure and luminescence property of $\mathrm{Gd}_{2} \mathrm{Si}_{2} \mathrm{O}_{7}$ on cerium concentration," 2007 IEEE Nuclear Science Symposium Conference Record, pp. 1426-1428.

13) Ludivine Pidol, Bruno Viana, Andreé Kahn-Harari, Auré lie Bessié re, Pieter Dorenbos, Nucl. Instr. and Meth. A 537 (2005) 125-129.

14) P. Dorenbos, J. Lumin. 91 (2000) 155.

15) C.W.E. van Eijk, P. Dorenbos and R. Visser, IEEE TRANSACIIONS ON NUCLEAR SCIENCE, VOL. 41, NO. 4, AUGUST 1994.

16) H. Ogino, A. Yoshikawa, M. Nikl, K. Kamada, T. Fukuda, Journal of Crystal Growth, Volume 292, Issue 2, 1 July 2006, 239-242. 